\title{
PPP1R12A Copy Number Is Associated with Clinical Outcomes of Stage III CRC Receiving Oxaliplatin-Based Chemotherapy
}

\author{
Chenbo Zhang, ${ }^{1}$ Ajian Li, ${ }^{1}$ Huaguang Li, ${ }^{2}$ Kangsheng Peng, ${ }^{3}$ Qing Wei, \\ Moubin Lin, ${ }^{2,5}$ Zhanju Liu, ${ }^{3}$ Lu Yin, ${ }^{5}$ and Jianwen $\mathrm{Li}^{1}$ \\ ${ }^{1}$ Department of General Surgery, Ruijin Hospital, Shanghai Jiaotong University School of Medicine, Shanghai 200025, China \\ ${ }^{2}$ Center for Translational Medicine, Yangpu Hospital Affiliated to Shanghai Tongji University School of Medicine, \\ Shanghai 200090, China \\ ${ }^{3}$ Department of Gastroenterology, The Tenth People's Hospital of Tongji University, Shanghai 200072, China \\ ${ }^{4}$ Department of Pathology, The Tenth People's Hospital of Tongji University, Shanghai 200072, China \\ ${ }^{5}$ Department of General Surgery, Yangpu Hospital Affiliated to Shanghai Tongji University School of Medicine, \\ Shanghai 200090, China \\ Correspondence should be addressed to Lu Yin; yinluipad@icloud.com and Jianwen Li; ljw5@yeah.net
}

Received 3 December 2014; Accepted 26 January 2015

Academic Editor: Vera L. Petricevich

Copyright (C) 2015 Chenbo Zhang et al. This is an open access article distributed under the Creative Commons Attribution License, which permits unrestricted use, distribution, and reproduction in any medium, provided the original work is properly cited.

\begin{abstract}
Aim. To investigate the correlation between PPP1R12A gene copy number and clinical outcomes of oxaliplatin-based regimen in stage III colorectal cancer (CRC). Methods. A total of 139 paraffin-embedded tissue samples of stage III CRC patients who received oxaliplatin-based treatment after radical surgery were recruited. Genomic DNA was extracted and purified from paraffin-embedded sections. Quantitative PCR methods were used to detect the relative copy number (RCN) of PPP1R12A. Results. Statistical analysis demonstrated that low PPP1R12A RCN was associated with poor RFS (HR $=2.186,95 \%$ CI: 1.293-3.696; $P=0.003$ ) and OS $(\mathrm{HR}=2.782,95 \% \mathrm{CI}: 1.531-5.052 ; P<0.001)$. Additionally, when patients were stratified according to subgroups of stage III and tumor location, poor RFS and OS were also observed in the low PPP1R12A RCN group with significance (RFS: IIIB HR = 2.870, $P<0.001$; colon HR $=1.910, P=0.037$; OS: IIIB HR $=3.527, P<0.001$; IIIC HR $=2.662, P=0.049$; rectum HR $=4.229$, $P=0.002)$. Conclusion. Our findings suggest the copy number of PPP1R12A can independently predict recurrence and overall survival of stage III colorectal cancer patients receiving oxaliplatin-based chemotherapy.
\end{abstract}

\section{Introduction}

Colorectal cancer (CRC) is the third most commonly diagnosed cancer in males and the second in females worldwide $[1,2]$ and the incidence rate and mortality rate attributed to CRC have been increasing in recent years in China. For the treatment of advanced CRC, chemotherapy is recommended and oxaliplatin-based regimens remain the first line chemotherapy in colorectal cancer. The recurrence-free survival of advanced CRC patients has been improved since the introduction of oxaliplatin-based chemotherapy treatment [3]. However, the chemoresistance remains a major obstacle and the treatment efficacy has reached a plateau $[4,5]$. Therefore, it is urgent to identify novel biomarkers capable of predicting the efficacy of oxaliplatin-based treatment [6].
PPP1R12A (protein phosphatase 1 regulatory subunit 12A) belongs to the myosin phosphatase targeting protein (MYPT) family and is also known as myosin phosphatase target subunit 1 (MYPT1). PPP1R12A molecule possesses a PP1cbinding motif, via which PPP1R12A recognizes and binds $\operatorname{PP1c} \delta$ (protein phosphatase 1, catalytic subunit, $\delta$ isoform) to form the PP1c $\delta$-MYPT1 complex. Through modulating the catalytic activity and specificity of PP1c $\delta$, PPP1R12A has been reported to participate in diverse cellular functions, such as smooth muscle contraction [7-9], cell migration and adhesion [10, 11], cell cycle regulation [12-14], and embryonic development regulation [15]. In addition, PPP1R12A possibly plays an important role in cancer chemoresistance and prognosis. Merlin, a tumor suppressor, is activated when the serine 


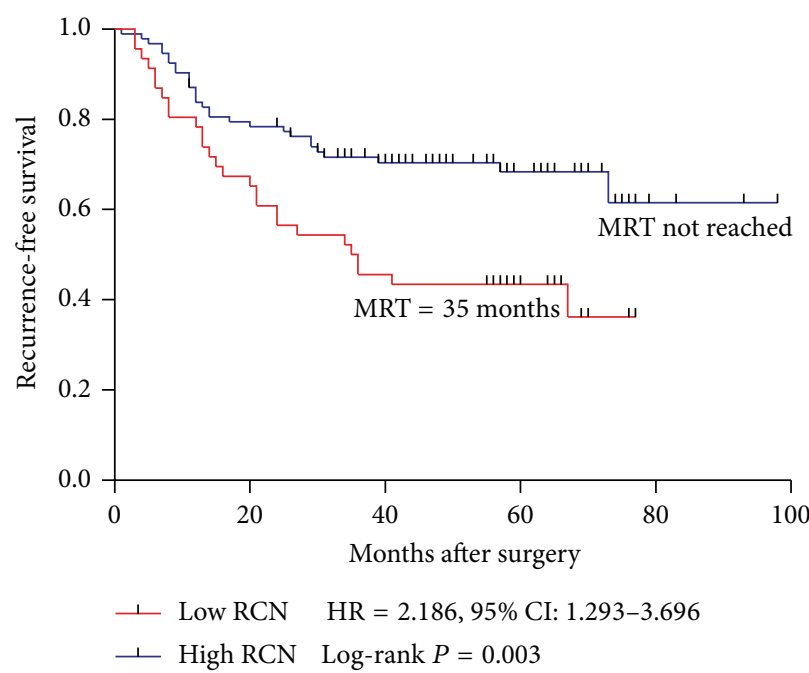

(a)

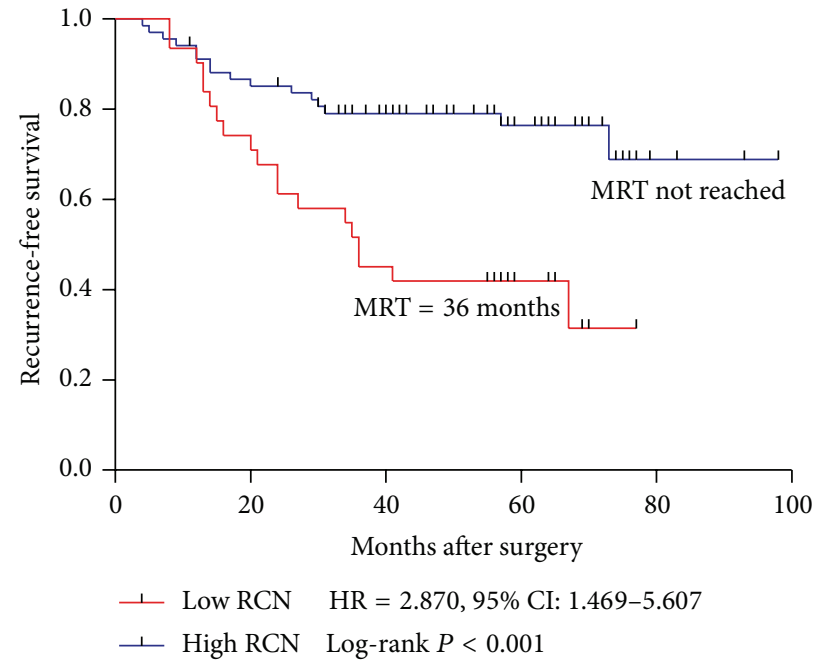

(b)

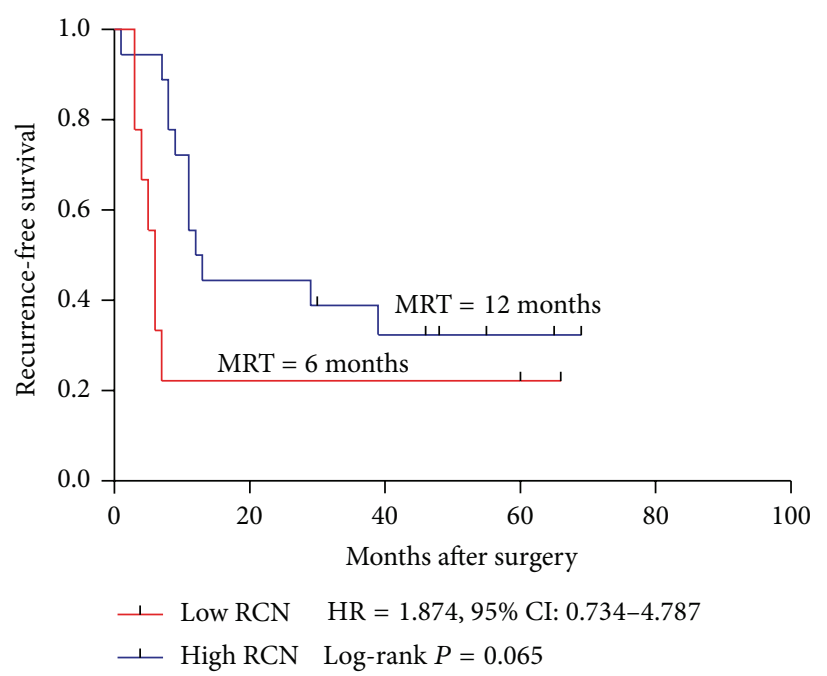

(c)

FIGURE 1: Kaplan-Meier survival curves of patients with stage III, IIIB, and IIIC disease receiving oxaliplatin-based chemotherapy. (a) RFS in total stage III; (b) RFS in stage IIIB; (c) RFS in stage IIIC. MRT: median recurrence time.

518 is dephosphorylated by the PPP1R12A-PP1c $\delta[16]$. Merlin loss leads to a coordinated increase of $\mathrm{Wnt} / \beta$-catenin signaling [17] and PI3K/AKT pathway [18], both of which promote chemoresistance in colorectal cancer $[19,20]$. Thus, we hypothesize that PPP1R12A associates with the recurrence and overall survival in colorectal cancer. However, so far there have been no studies exploring the correlation between PPP1R12A and clinical outcomes in colorectal cancer.

In this study, we employed the quantitative PCR to detect the relative copy number (RCN) of PPP1R12A in stage III CRC patients receiving oxaliplatin-based regimen and compute the association of PPP1R12A gene copy number with clinical outcomes.

\section{Materials and Methods}

2.1. Human Subjects. This study included a total of 139 paraffin-embedded tissue samples of the patients who received curative resection from 2006 to 2011 in the Ruijin Hospital affiliated to Shanghai Jiao Tong University and the Tenth People's Hospital affiliated to Shanghai Tongji University. All the patients were histologically and clinically diagnosed as the stage III of CRC according to the criteria proposed by the Standard American Joint Committee on Cancer (AJCC) and only patients receiving at least 6 cycles of oxaliplatinbased chemotherapy after operation were included in this study. None of the patients received chemotherapy or radiotherapy before radical surgery. Written informed consent was obtained from all participants. This study was approved by the Medical Ethical Committee of Shanghai Jiaotong University and Tongji University.

2.2. DNA Extraction. Genomic DNA was extracted and purified from $5 \mu \mathrm{m}$ thick formalin-fixed paraffin-embedded sections (tumor cells account for at least 70\%) of the 139 tumors 
TABLE 1: Selected demographic and clinical characteristics of patients with CRCs.

\begin{tabular}{|c|c|c|c|c|c|}
\hline Clinicopathological factors & $\begin{array}{c}\text { Number of patients } \\
\qquad N=139\end{array}$ & $\begin{array}{c}\text { Recurrence } \\
\text { Number (\%) }\end{array}$ & $P$ value & $\begin{array}{c}\text { Dead } \\
\text { Number (\%) }\end{array}$ & $P$ value \\
\hline Age (years) & & 56 & & 44 & \\
\hline$\geq 65$ & $53(38.1 \%)$ & $22 / 53(41.5 \%)$ & 0.818 & $19 / 53(35.8 \%)$ & 0.404 \\
\hline$<65$ & $86(61.9 \%)$ & $34 / 86(39.5 \%)$ & & $25 / 86(29.1 \%)$ & \\
\hline \multicolumn{6}{|l|}{ Sex } \\
\hline Male & $85(61.2 \%)$ & $33 / 85(38.8 \%)$ & 0.659 & $25 / 85(29.4 \%)$ & 0.476 \\
\hline Female & $54(38.8 \%)$ & $23 / 54(42.6 \%)$ & & $19 / 54(35.2 \%)$ & \\
\hline \multicolumn{6}{|l|}{ Tumor site } \\
\hline Rectum & $67(48.2 \%)$ & $26 / 67(38.8 \%)$ & 0.731 & $19 / 67(28.4 \%)$ & 0.42 \\
\hline Colon & $72(51.8 \%)$ & $30 / 72(41.7 \%)$ & & $25 / 72(34.7 \%)$ & \\
\hline \multicolumn{6}{|l|}{ Histology } \\
\hline Well/moderate & $113(81.3 \%)$ & $43 / 113(38.1 \%)$ & 0.263 & $31 / 113(27.4 \%)$ & 0.026 \\
\hline Poor & $26(18.7 \%)$ & $13 / 26(50.0 \%)$ & & $13 / 26(50.0 \%)$ & \\
\hline \multicolumn{6}{|l|}{ Lymph node metastasis } \\
\hline$\geq 4$ & $31(22.3 \%)$ & $18 / 31(58.1 \%)$ & 0.022 & $17 / 31(54.8 \%)$ & 0.002 \\
\hline$<4$ & $108(77.7 \%)$ & $38 / 108(35.2 \%)$ & & $27 / 108(25.0 \%)$ & \\
\hline \multicolumn{6}{|l|}{ TNM stage } \\
\hline IIIA & $13(9.4 \%)$ & $2 / 13(15.4 \%)$ & 0.001 & $1 / 13(7.7 \%)$ & 0.004 \\
\hline IIIB & $99(71.2 \%)$ & $35 / 99(35.4 \%)$ & & $28 / 99(28.3 \%)$ & \\
\hline IIIC & $27(19.4 \%)$ & $19 / 27(70.4 \%)$ & & $15 / 27(55.6 \%)$ & \\
\hline
\end{tabular}

Bold items highlight $P<0.05$; TNM: tumor node metastasis.

using a DNeasy kit (Qiagen, CA, USA) according to the manufacturer's instructions.

2.3. Real-Time Quantitative PCR. To detect PPP1R12A gene DNA copy number, real-time quantitative polymerase chain reaction (qPCR) was performed using IQ5 Multicolour RealTime PCR Detection system (Bio-Rad, CA, USA). qPCR was carried out using the SYBR Green kit (Qiagen, Germany) in a final volume of $20 \mu \mathrm{L}$ with housekeeping gene glyceraldehyde 3-phosphate dehydrogenase (GAPDH) used as an internal control. The primers for GAPDH were forward, $5^{\prime}$-ACG AAT TTG GCT ACA GCA ACA GG-3', and reverse, $5^{\prime}$-CCA GCA GTG AGG GTC TCT CTC TT-3'. The primers for PPP1R12A were forward, 5'-AGG TGA AGT TCG ACG ATG GC-3', and reverse, $5^{\prime}$-TCC GTC CAC ATT GGC GTA AT-3'. Each thermal cycle consisted of $15 \mathrm{~s}$ of denaturation at $95^{\circ} \mathrm{C}, 20 \mathrm{~s}$ of primer annealing at $60^{\circ} \mathrm{C}$, and $20 \mathrm{~s}$ of extension at $72^{\circ} \mathrm{C}$. All the samples (both PPP1R12A and GAPDH) were run in triplicate.

2.4. Statistical Analysis. Each assay was tested in triplicate. Data points that generated triplicate $\mathrm{Ct}$ values with over one cycle variance were excluded from analysis. Since the samples were analyzed in triplicate, values for each triplicate sample were averaged to the final data use. The mean Ct value obtained from each sample was normalized to the averaged copy number and then subjected to analysis with the $2^{-\Delta \Delta \mathrm{Ct}}$ method $[21,22]$.

We performed the Pearson Chi-square $\left(\chi^{2}\right)$ to compare the distributions of categorical variables. Recurrence-free survival (RFS) was calculated from the surgery date to the date of disease recurrence or death. Overall survival (OS) was defined as the time from the date of surgery to that of death or the end of follow-up. We dichotomized patients into high RCN and low RCN groups at median, tertile and quartile cutoff points, and it showed that the most significant associations of PPP1R12A RCN with RFS and OS were achieved at first tertile point (Supplemental Tables 1 and 2, in Supplementary Material available online at http://dx.doi.org/10.1155/2015/ 417184). So we chose the first tertile of PPP1R12A copy number as the cut-off point in our study [23]. Hazard ratios (HRs) and $95 \%$ confidence intervals (CIs) were estimated using Kaplan-Meier analysis, as well as multivariate COX regression, while adjusting for age, location, and gender. All statistical tests were two-sided. Statistical analysis was performed using the SPSS Software, version 20.0 (Chicago, IL, United States). $P<0.05$ was considered statistically significant.

\section{Result}

3.1. Patient Characteristics. The demographic and clinical characteristics in the 139 patients with CRCs were summarized in Table 1. The patient pool comprised eighty-five (61.2\%) males and fifty-four (38.8\%) females. Of the 139 patients, the median age was 61 years (range, 25-84) with the mean age of 61.06 years. The tumors were found in the colon $(n=72,51.8 \%)$ and rectum $(n=67,48.2 \%)$. The patients with stage IIIA, IIIB, and IIIC disease accounted for $9.4 \%(n=13)$, $71.2 \%(n=99)$, and $19.4 \%(n=27)$, respectively. After a median follow-up of 49 months (range, 4-98 months), 56 patients (40.3\%) exhibited tumor recurrence and 44 patients (31.7\%) died. 
TABLE 2: Univariate and multivariate analyses for recurrence-free survival (Cox proportional hazard model).

\begin{tabular}{|c|c|c|c|c|c|c|}
\hline \multirow{2}{*}{ Variable } & \multicolumn{3}{|c|}{ Univariate analysis } & \multicolumn{3}{|c|}{ Multivariate analysis } \\
\hline & $\mathrm{HR}$ & $95 \% \mathrm{CI}$ & $P$ value & HR & $95 \% \mathrm{CI}$ & $P$ value \\
\hline Age ( $\geq 65$ versus $<65$ year old) & 1.116 & $0.652-1.908$ & 0.689 & & & \\
\hline Sex (male versus female) & 0.844 & $0.495-1.441$ & 0.535 & & & \\
\hline \multicolumn{7}{|l|}{ Tumor site } \\
\hline Colon versus rectum & 1.075 & $0.635-1.821$ & 0.788 & & & \\
\hline \multicolumn{7}{|l|}{ Histology } \\
\hline Poor versus well/moderate & 1.548 & $0.832-2.881$ & 0.168 & & & \\
\hline \multicolumn{7}{|l|}{ Lymph node metastasis } \\
\hline$n \geq 4$ versus $n<4$ & 2.160 & $1.230-3.793$ & 0.007 & 0.832 & $0.409-1.693$ & 0.612 \\
\hline \multicolumn{7}{|l|}{ TNM stage } \\
\hline IIIC versus IIIA + IIIB & 4.001 & $2.281-7.017$ & $<0.001$ & 5.057 & $2.478-10.323$ & $<0.001$ \\
\hline PPP1R12A (low versus high) & 2.186 & $1.293-3.696$ & 0.003 & 2.596 & $1.500-4.490$ & 0.001 \\
\hline
\end{tabular}

Bold items highlight $P<0.05$, HR: hazard ratio, CI: confidence interval; TNM: tumor node metastasis.

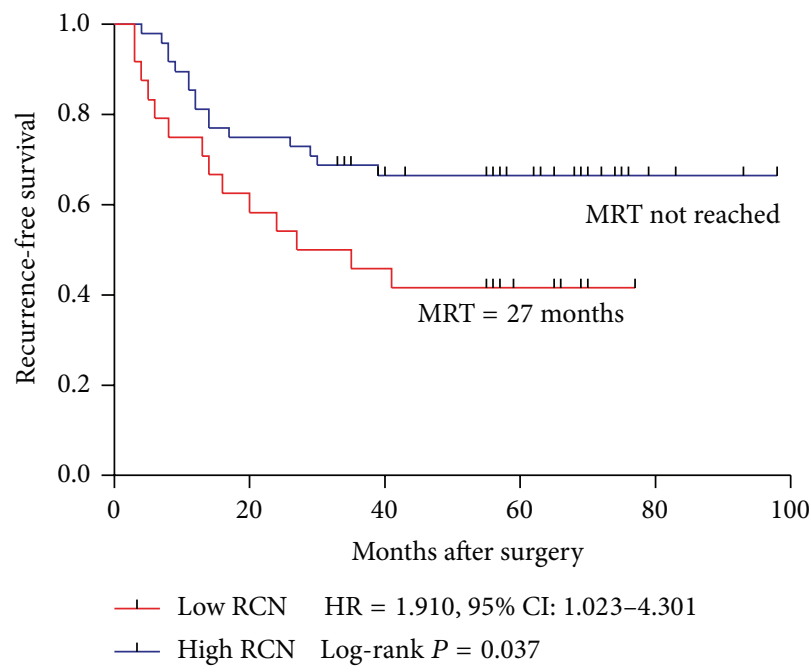

(a)

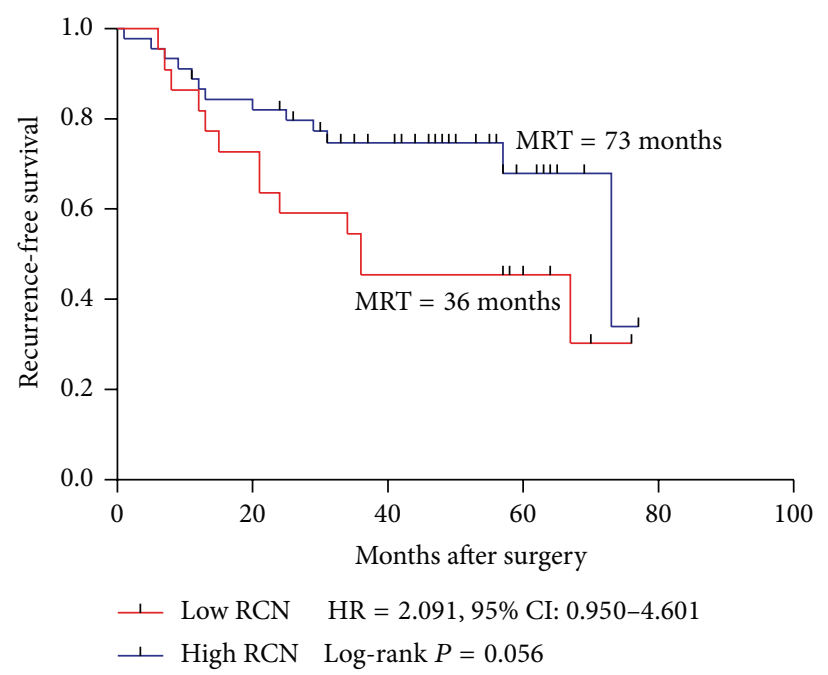

(b)

FIGURE 2: Kaplan-Meier survival curves of patients with stage III, IIIB, and IIIC disease receiving oxaliplatin-based chemotherapy. (a) RFS in colon; (b) RFS in rectum. MRT: median recurrence time.

3.2. Correlation between PPP1R12A Copy Number and Recurrence-Free Survival. Patients were dichotomized into high $\mathrm{RCN}(\mathrm{RCN} \geq 0.37, n=93)$ and low $\mathrm{RCN}(\mathrm{RCN}<0.37$, $n=46)$ groups with a tertile cut-off of PPP1R12A relative copy number $(\mathrm{RCN}=0.37)$. During the follow-up, $56(40.3 \%)$ of the 139 patients suffered recurrence (Table 1). The median recurrence-free periods were $35( \pm 9.6)$ months in the low RCN group while the median RFS were not reached in the high RCN group (Figure 1(a)). Both the univariate analysis and the multivariate analysis of RFS were shown in Table 2. High lymph node metastasis (lymph node $\geq 4$ : HR $=2.160$, $P=0.007$ ), high TNM stage (IIIC: $\mathrm{HR}=4.001, P<0.001$ ), and low RCN of PPP1R12A ( RCN < 0.37: HR = 2.186, $P=$ 0.003 ) were significantly associated with poor RFS. After adjusting for age, location, gender, TNM, lymph node metastasis, and histology grade, PPP1R12A remained an independent factor associated with RFS. In the Kaplan-Meier analysis, the curves showed that patients with low PPP1R12A copy number were significantly associated with poor RFS $(P=$ 0.003) (Figure 1(a)). To investigate the correlation between PPP1R12A and RFS in subgroups of patients, we performed RFS analysis among IIIB, IIIC, colon cancer, and rectal cancer, respectively. The small size of IIIA patients remained the limitation towards further analysis. Patients with low RCN of PPP1R12A showed poorer RFS than high RCN patients with high significance in IIIB $(\mathrm{HR}=2.870, P<0.001)$ (Figure 1(b)). Also, low RCN of PPP1R12A was associated with poor RFS in colon cancer with significance $(P=0.037)$ (Figure 2(a)). In the IIIC group and rectum group, patients with low PPP1R12A RCN showed a trend toward poor RFS (IIIC: $\mathrm{HR}=1.874, P=0.065$, rectum $\mathrm{HR}=2.091, P=0.056$ ) (Figures 1(c) and 2(b)).

3.3. Correlation between PPP1R12A Copy Number and Overall Survival. Among the 139 stage III CRC patients, 44 (31.7\%) 


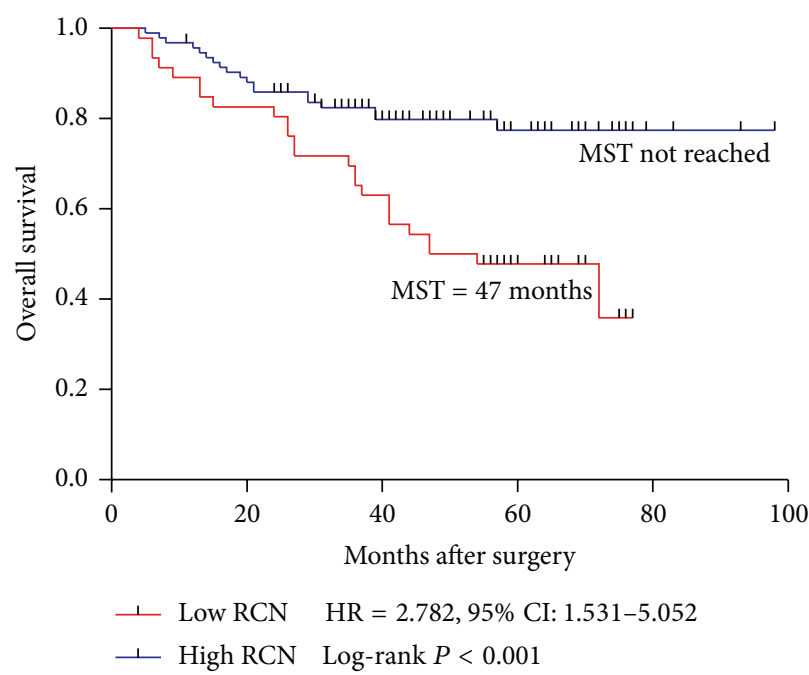

(a)

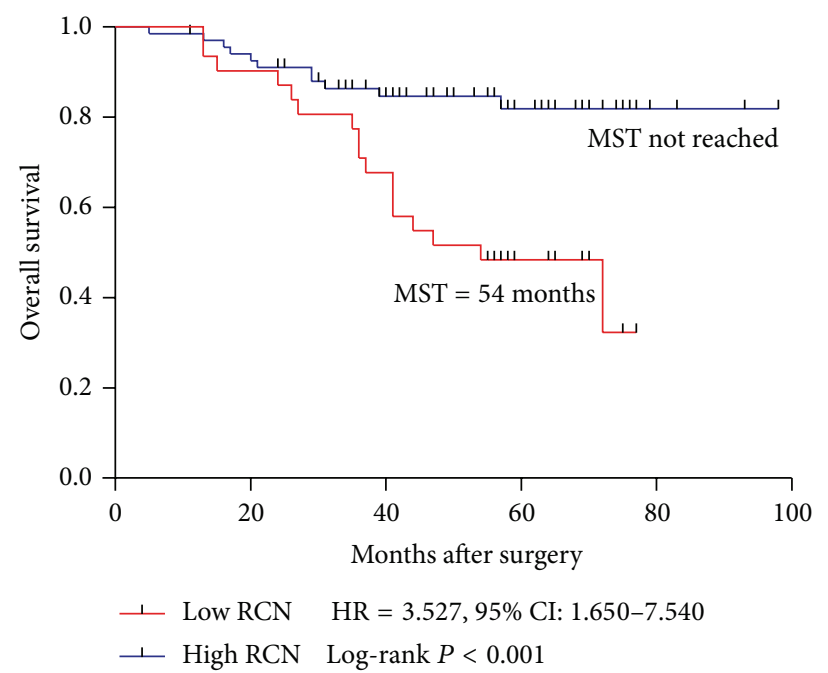

(b)

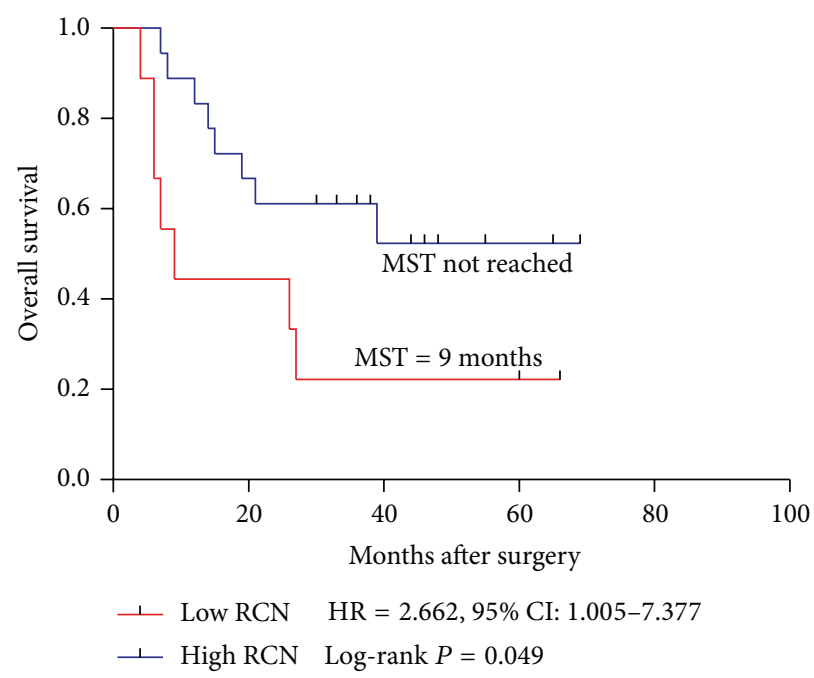

(c)

FIgURE 3: Kaplan-Meier survival curves of patients with stage III, IIIB, and IIIC disease receiving oxaliplatin-based chemotherapy. (a) OS in total stage III; (b) OS in stage IIIB; (c) OS in stage IIIC. MST: median survival time.

patients died during the follow-up. The median overall periods were $47( \pm 11.1)$ months in the low RCN group while the OS were not reached in the high gene copy number group (Figure 3(a)). Both the univariate analysis and the multivariate analysis of OS were shown in Table 3. Poor differentiation $(\mathrm{HR}=2.310, P=0.011)$, high lymph node metastasis (lymph node $\geq 4: \mathrm{HR}=2.750, P=0.001$ ), high TNM stage (IIIC: $\mathrm{HR}=3.613, P<0.001)$, and low PPP1R12A RCN $(\mathrm{RCN}<$ $0.37: \mathrm{HR}=2.782, P<0.001$ ) were shown to be significantly associated with poor OS. Furthermore, the multivariate analysis showed that poor differentiation $(\mathrm{HR}=2.242, P=$ 0.021 ), TNM stage (IIIC: $\mathrm{HR}=3.913, P<0.001$ ), and low PPP1R12A $(\mathrm{HR}=2.976, P=0.001)$ remained independent predictive factors of poor OS. The Kaplan-Meier analysis was also used to assess the correlation between PPP1R12A copy number and overall survival. The log-rank test showed that patients with low PPP1R12A copy number were associated with a statistically significant poor OS $(P<0.001)$
(Figure 3(a)). Further analysis in different subgroups showed low RCN was still associated with OS in IIIB (IIIB: $P<0.001$, $\mathrm{HR}=3.527$ ) and IIIC (IIIC: $P=0.049, \mathrm{HR}=2.662$ ) patients (Figures 3(b) and 3(c)). In the rectum subgroup, low RCN was significantly correlated with poor OS $(P=0.002, \mathrm{HR}=$ 4.229) (Figure 4(b)). Similarly, patients with low PPP1R12A RCN showed a trend towards poor OS in colon subgroup (Figure 4(a) $P=0.086, \mathrm{HR}=1.957$ ).

\section{Discussion}

Colorectal cancer remains one of the most prevalent cancer and leading causes of cancer deaths worldwide. Adjuvant chemotherapy is thus required to reduce relapse rate and enhance survival. Oxaliplatin-based regimen is widely used as first-line therapy for advanced colorectal cancer; however, the recurrence is still high. So identification of patients who 
TABLE 3: Univariate and multivariate analyses for overall survival (Cox proportional hazard model).

\begin{tabular}{|c|c|c|c|c|c|c|}
\hline \multirow{2}{*}{ Variable } & \multicolumn{3}{|c|}{ Univariate analysis } & \multicolumn{3}{|c|}{ Multivariate analysis } \\
\hline & HR & $95 \% \mathrm{CI}$ & $P$ value & HR & $95 \% \mathrm{CI}$ & $P$ value \\
\hline Age ( $\geq 65$ versus $<65$ year old) & 1.301 & $0.716-2.363$ & 0.387 & & & \\
\hline Sex (male versus female) & 0.754 & $0.414-1.372$ & 0.355 & & & \\
\hline \multicolumn{7}{|l|}{ Tumor site } \\
\hline Colon versus rectum & 1.202 & $0.661-2.187$ & 0.546 & & & \\
\hline \multicolumn{7}{|l|}{ Histology } \\
\hline Poor versus well/moderate & 2.310 & $1.208-4.418$ & 0.011 & 2.242 & $1.128-4.455$ & 0.021 \\
\hline \multicolumn{7}{|l|}{ Lymph node metastasis } \\
\hline$n \geq 4$ versus $n<4$ & 2.750 & $1.497-5.050$ & 0.001 & 1.050 & $0.492-2.243$ & 0.899 \\
\hline \multicolumn{7}{|l|}{ TNM stage } \\
\hline IIIC versus IIIA + IIIB & 3.613 & $1.919-6.803$ & $<0.001$ & 3.913 & $1.831-8.366$ & $<0.001$ \\
\hline PPP1R12A (low versus high) & 2.782 & $1.531-5.052$ & $<0.001$ & 2.976 & $1.602-5.528$ & 0.001 \\
\hline
\end{tabular}

Bold items highlight $P<0.05$; HR: hazard ratio; CI: confidence interval; TNM: tumor node metastasis.

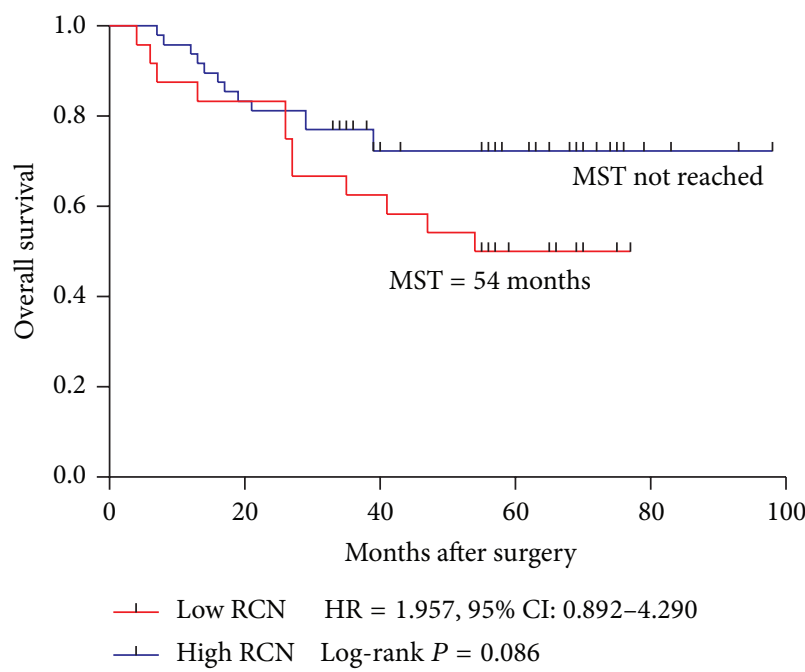

(a)

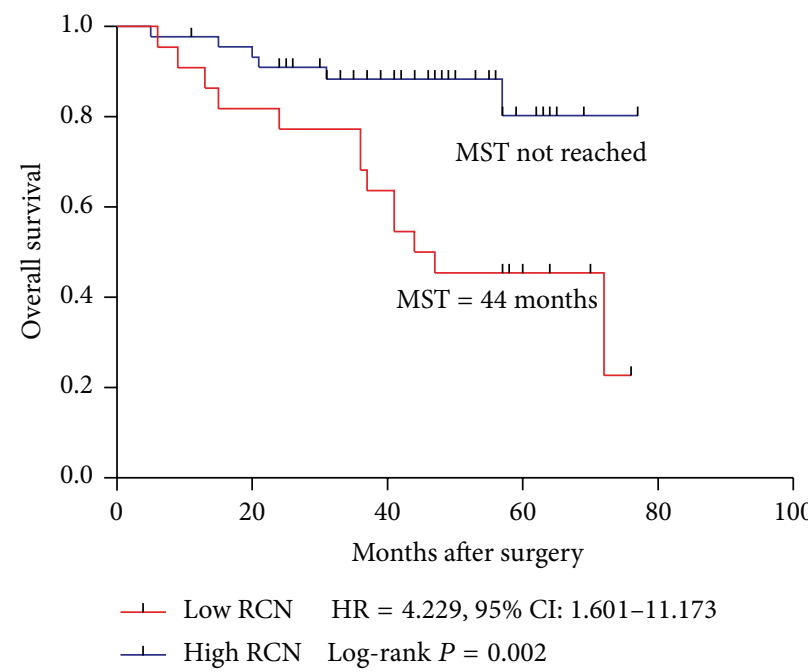

(b)

FIGURE 4: Kaplan-Meier survival curves of patients with stage III, IIIB, and IIIC disease receiving oxaliplatin-based chemotherapy. (a) OS in colon; (b) OS in rectum. MST: median survival time.

will likely or unlikely benefit from chemotherapy would allow personalized treatment of CRC patients.

Here for the first time we conducted the study to examine the possibility of PPP1R12A as a possible biomarker for the prediction of clinical outcomes in stage III colorectal cancer patients treated with oxaliplatin-based chemotherapy. The results showed that patients with low copy number of PPP1R12A had a poor OS and RFS with statistical significance, indicating PPP1R12A was able to predict recurrence and overall survival. PPP1R12A is essential in the activation of Merlin [16]. Serine 518 of Merlin is dephosphorylated by phosphatase (PPP1R12A-PP1c). And the dephosphorylated merlin exerts inhibitory effect on downstream signaling pathways, for example, PI3K/Akt and Wnt/ $\beta$-catenin signaling $[17,18]$. Recent studies have demonstrated that inhibition of PI3K would lead to increased oxaliplatin-induced cell apoptosis and increased oxaliplatin sensitivity in cholangiocarcinoma cells [24]. In addition, Wnt/ $\beta$-catenin pathway has been revealed to enhance chemoresistance in many cancer cell types including hepatocellular carcinoma and neuroblastoma and this kind of chemoresistance is thought to be associated with overexpression of multidrug resistance 1 gene (MDR1) $[25,26]$. Probably PPP1R12A activates Merlin which represses $\mathrm{PI} 3 \mathrm{~K} / \mathrm{Akt}$ and $\mathrm{Wnt} / \beta$-catenin signaling and leads to inhibition of drug resistance and enhances the apoptosis induced by oxaliplatin.

Conventional TNM staging system is not able to predict the risk of recurrence among certain subgroup patients such as IIIB and IIIC. In this study, we showed that PPP1R12A RCN was significantly correlated with OS and RFS even in IIIB and IIIC. This indicates PPP1R12A can not only predict OS and RFS of stage III CRC patients, but also predict the OS and RFS of IIIB and IIIC patients. This compensates the weakness of TNM staging and allows clinicians to make the more accurate therapeutic decision. 
In conclusion, our study demonstrates that the copy number of PPP1R12A could independently predict recurrence and overall survival of stage III colorectal cancer patients receiving oxaliplatin-based adjuvant chemotherapy.

\section{Conflict of Interests}

The authors declare that there is no conflict of interests regarding the publication of this paper.

\section{Acknowledgments}

This work was supported by the grant from National Natural Science Foundation of China (81272480) and Natural Science Foundation of Shanghai (11DZ1910200, 12ZR1418800, and 11nm0503800).

\section{References}

[1] A. Jemal, F. Bray, M. M. Center, J. Ferlay, E. Ward, and D. Forman, "Global cancer statistics," CA: A Cancer Journal for Clinicians, vol. 61, no. 2, pp. 69-90, 2011.

[2] J. Ferlay, H.-R. Shin, F. Bray, D. Forman, C. Mathers, and D. M. Parkin, "Estimates of worldwide burden of cancer in 2008: GLOBOCAN 2008," International Journal of Cancer, vol. 127, no. 12, pp. 2893-2917, 2010.

[3] S. Giacchetti, B. Perpoint, R. Zidani et al., "Phase III multicenter randomized trial of oxaliplatin added to chronomodulated fluorouracil-leucovorin as first-line treatment of metastatic colorectal cancer," Journal of Clinical Oncology, vol. 18, no. 1, pp. 136-147, 2000.

[4] L. Kelland, "The resurgence of platinum-based cancer chemotherapy," Nature Reviews Cancer, vol. 7, no. 8, pp. 573-584, 2007.

[5] R. M. Goldberg, D. J. Sargent, R. F. Morton et al., "A randomized controlled trial of fluorouracil plus leucovorin, irinotecan, and oxaliplatin combinations in patients with previously untreated metastatic colorectal cancer," Journal of Clinical Oncology, vol. 22, no. 1, pp. 23-30, 2004.

[6] J. S. Ross, J. Torres-Mora, N. Wagle, T. A. Jennings, and D. M. Jones, "Biomarker-based prediction of response to therapy for colorectal cancer: current perspective," The American Journal of Clinical Pathology, vol. 134, no. 3, pp. 478-490, 2010.

[7] D. P. Wilson, M. Susnjar, E. Kiss, C. Sutherland, and M. P. Walsh, "Thromboxane $\mathrm{A}_{2}$-induced contraction of rat caudal arterial smooth muscle involves activation of $\mathrm{Ca}^{2+}$ entry and $\mathrm{Ca}^{2+}$ sensitization: Rho-associated kinase-mediated phosphorylation of MYPT1 at Thr-855, but not Thr-697," Biochemical Journal, vol. 389, no. 3, pp. 763-774, 2005.

[8] F. Matsumura and D. J. Hartshorne, "Myosin phosphatase target subunit: many roles in cell function," Biochemical and Biophysical Research Communications, vol. 369, no. 1, pp. 149-156, 2008.

[9] M. Ito, T. Nakano, F. Erdodi, and D. J. Hartshorne, "Myosin phosphatase: structure, regulation and function," Molecular and Cellular Biochemistry, vol. 259, no. 1-2, pp. 197-209, 2004.

[10] D. Xia, J. T. Stull, and K. E. Kamm, "Myosin phosphatase targeting subunit 1 affects cell migration by regulating myosin phosphorylation and actin assembly," Experimental Cell Research, vol. 304, no. 2, pp. 506-517, 2005.

[11] M. E. Grassie, L. D. Moffat, M. P. Walsh, and J. A. MacDonald, "The myosin phosphatase targeting protein (MYPT) family: a regulated mechanism for achieving substrate specificity of the catalytic subunit of protein phosphatase type 18," Archives of Biochemistry and Biophysics, vol. 510, no. 2, pp. 147-159, 2011.

[12] H.-S. Cho, T. Suzuki, N. Dohmae et al., "Demethylation of RB regulator MYPT1 by histone demethylase LSD1 promotes cell cycle progression in cancer cells," Cancer Research, vol. 71, no. 3, pp. 655-660, 2011.

[13] J. Li, X. Liu, J. Liao et al., "MYPT1 sustains centromeric cohesion and the spindle-assembly checkpoint," Journal of Genetics and Genomics, vol. 40, no. 11, pp. 575-578, 2013.

[14] T. Chiyoda, N. Sugiyama, T. Shimizu et al., "LATS1/WARTS phosphorylates MYPT1 to counteract PLK1 and regulate mammalian mitotic progression," The Journal of Cell Biology, vol. 197, no. 5, pp. 625-641, 2012.

[15] R. Okamoto, M. Ito, N. Suzuki et al., "The targeted disruption of the MYPT1 gene results in embryonic lethality," Transgenic Research, vol. 14, no. 3, pp. 337-340, 2005.

[16] H. Jin, T. Sperka, P. Herrlich, and H. Morrison, “Tumorigenic transformation by CPI-17 through inhibition of a merlin phosphatase," Nature, vol. 442, no. 7102, pp. 576-579, 2006.

[17] L. Zhou, E. Ercolano, S. Ammoun, M. C. Schmid, M. A. Barczyk, and C. O. Hanemann, "Merlin-deficient human tumors show loss of contact inhibition and activation of Wnt/beta-catenin signaling linked to the PDGFR/Src and Rac/PAK pathways," Neoplasia, vol. 13, no. 12, pp. 1101-1112, 2011.

[18] R. Rong, X. Tang, D. H. Gutmann, and K. Ye, "Neurofibromatosis 2 (NF2) tumor suppressor merlin inhibits phosphatidylinositol 3-kinase through binding to PIKE-L," Proceedings of the National Academy of Sciences of the United States of America, vol. 101, no. 52, pp. 18200-18205, 2004.

[19] M. Bordonaro, S. Tewari, C. E. Cicco, W. Atamna, and D. L. Lazarova, "A switch from canonical to noncanonical wnt signaling mediates drug resistance in colon cancer cells," PLoS ONE, vol. 6, no. 11, Article ID e27308, 2011.

[20] P. Michl and J. Downward, "Mechanisms of disease: PI3K/AKT signaling in gastrointestinal cancers," Zeitschrift für Gastroenterologie, vol. 43, no. 10, pp. 1133-1139, 2005.

[21] K. J. Livak and T. D. Schmittgen, "Analysis of relative gene expression data using real-time quantitative PCR and the 2$\Delta \Delta$ CT method," Methods, vol. 25, no. 4, pp. 402-408, 2001.

[22] K. Kasem, V. Gopalan, A. Salajegheh, C.-T. Lu, R. A. Smith, and A. K.-Y. Lam, "JK1 (FAM134B) gene and colorectal cancer: a pilot study on the gene copy number alterations and correlations with clinicopathological parameters," Experimental and Molecular Pathology, vol. 97, no. 1, pp. 31-36, 2014.

[23] E. Dotan, N. J. Meropol, F. Zhu et al., "Relationship of increased aurora kinase A gene copy number, prognosis and response to chemotherapy in patients with metastatic colorectal cancer," British Journal of Cancer, vol. 106, no. 4, pp. 748-755, 2012.

[24] K. Leelawat, S. Narong, W. Udomchaiprasertkul, S. Leelawat, and S. Tungpradubkul, "Inhibition of PI3K increases oxaliplatin sensitivity in cholangiocarcinoma cells," Cancer Cell International, vol. 9, article 3, 2009.

[25] T. Noda, H. Nagano, I. Takemasa et al., "Activation of Wnt/ $\beta$ catenin signalling pathway induces chemoresistance to interferon- $\alpha / 5$-fluorouracil combination therapy for hepatocellular carcinoma," British Journal of Cancer, vol. 100, no. 10, pp. 16471658, 2009.

[26] M. Flahaut, R. Meier, A. Coulon et al., “The Wnt receptor FZD1 mediates chemoresistance in neuroblastoma through activation of the Wnt/ $\beta$-catenin pathway," Oncogene, vol. 28, no. 23, pp. 2245-2256, 2009. 


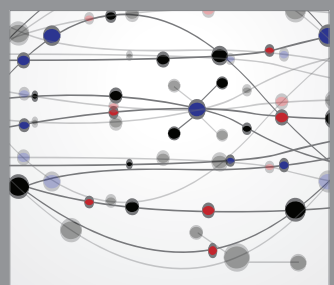

The Scientific World Journal
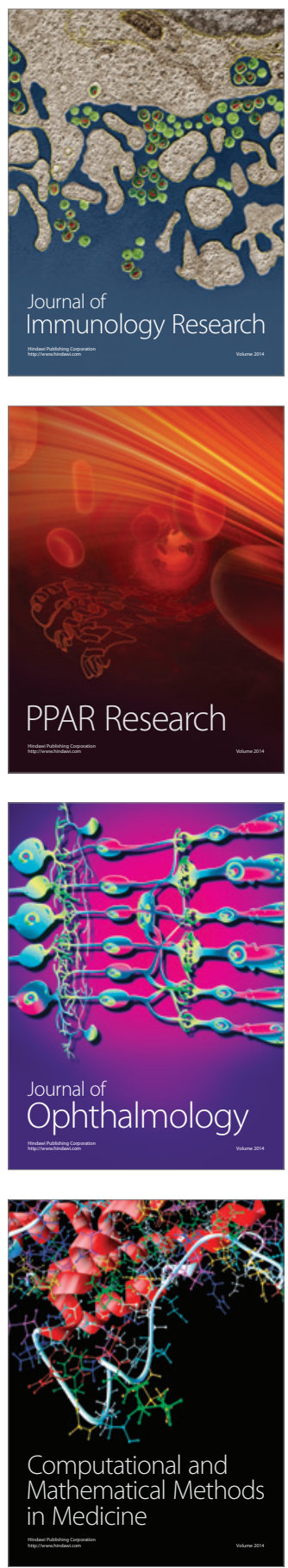

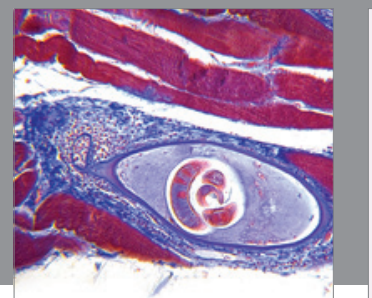

Gastroenterology

Research and Practice
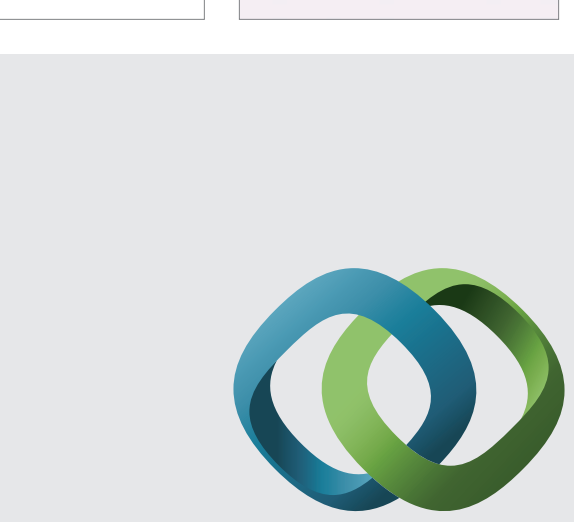

\section{Hindawi}

Submit your manuscripts at

http://www.hindawi.com
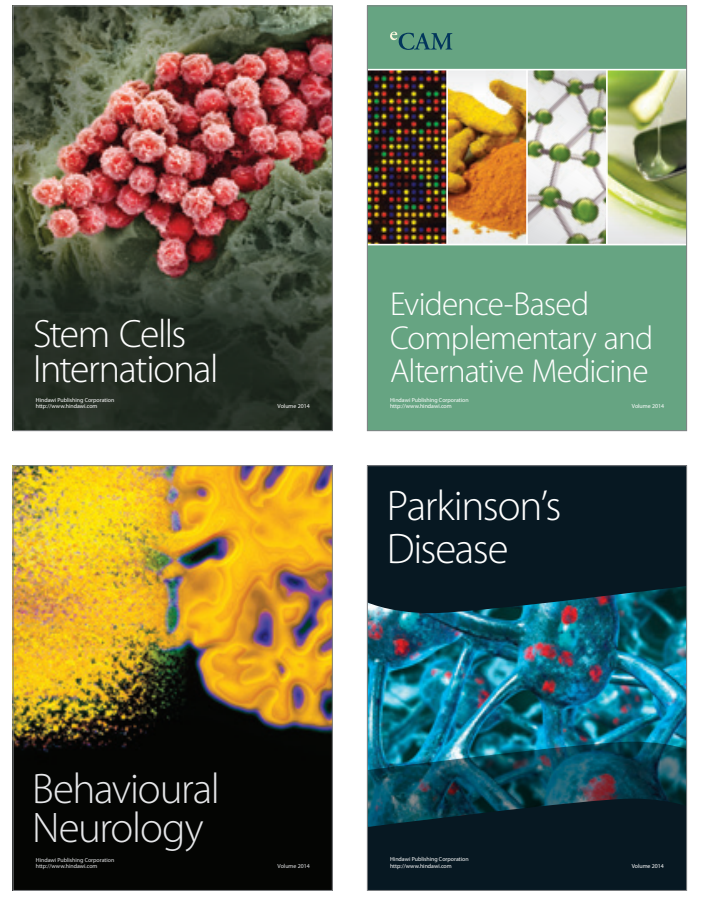
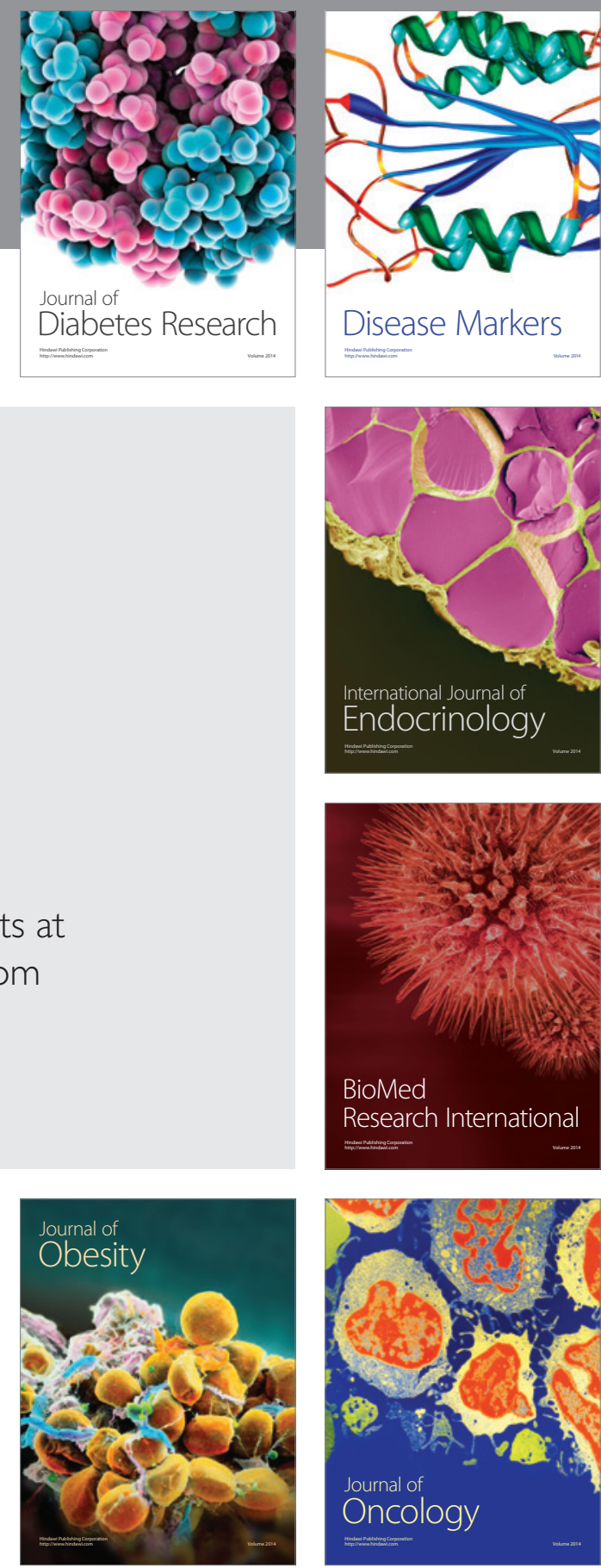

Disease Markers
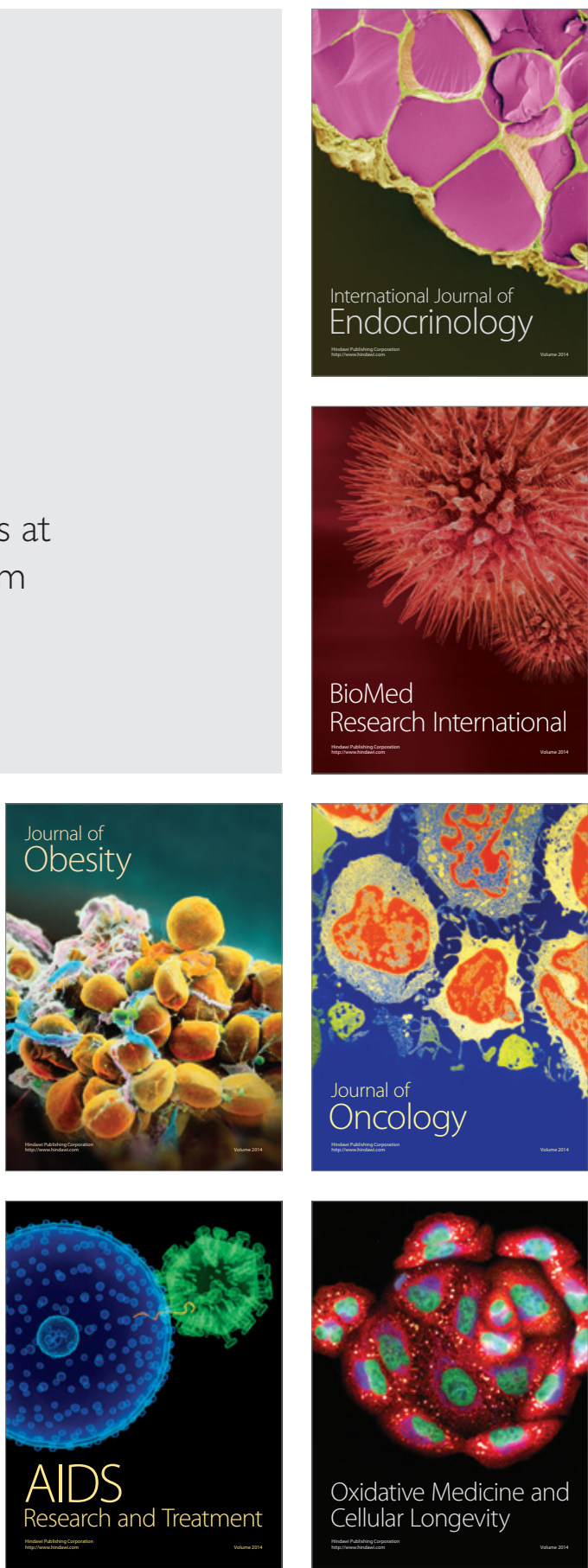